\title{
Ultrasonography for long-term evaluation of hyaluronic acid filler in the face: A technical report of 180 days of follow-up
}

\author{
Luiz Paulo Carvalho Rocha $\mathbb{D}^{1}$, Tânia de Carvalho Rocha $\mathbb{D}^{2}$, Stephanie de Cássia Carvalho Rocha $\mathbb{D}^{2}$,

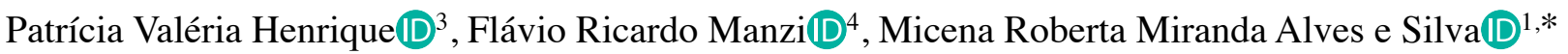 \\ ${ }^{1}$ Department of Morphology, Institute of Biological Sciences, Universidade Federal de Minas Gerais, Belo Horizonte, Brazil \\ ${ }^{2}$ Hermes Pardini Group, Belo Horizonte, Brazil \\ ${ }^{3}$ Nucleodonto, Brasília, Brazil \\ ${ }^{4}$ Department of Dentistry, Oral Radiology, Pontifical Catholic University of Minas Gerais, Belo Horizonte, Brazil
}

\section{ABSTRACT}

Purpose: In this study, we used ultrasonography to monitor the use of hyaluronic acid (HA) as a filler in the face for esthetic reasons. We monitored changes in the filler shape, distribution, and relationship with adjacent anatomical structures over a 180-day period.

Materials and Methods: Two patients each received an ultrasound-guided injection of HA, with different products and application sites for each patient. In 1 patient, the injection was administered in the angle of the mandible, while in the other, it was administered in the zygomatic region. The injection sites were monitored via ultrasonography at 24 hours, 30 days, and 180 days, at which times the imaging characteristics of the filler were observed. All injections were performed by the same professional, as were the ultrasound exams, which were conducted using the same equipment.

Results: In both cases, the HA fillers were visualized using ultrasound at all time points. Some differences were observed between the cases in the images and the distribution of the pockets of filler. In 1 case, the filler appeared as a dark hypoechoic region with well-defined contours, and the material was observed to have moved posteriorly by the 180-day mark. In the other case, the material appeared hyperechoic relative to the previous case and presented no noticeable changes in its anteroposterior distribution over time.

Conclusion: Based on these 2 cases, ultrasonography can be a complementary tool used to monitor facial fillers over the long term, allowing for the dynamic observation of different fillers.(Imaging Sci Dent 2020; 50: 175-80)

KEY WORDS: Ultrasonography; Esthetics; Hyaluronic Acid; Dermal Fillers

\section{Introduction}

Ultrasonography (US) is a high-resolution imaging technique that is specific for soft-tissue evaluation and that can provide a wealth of information before, during, and after clinical procedures. While US of the facial region has not been extensively explored, it can provide important details to assist in the planning and execution of procedures. ${ }^{1}$ To evaluate the facial region, which is anatomically intricate, contains many tissue types, and has shallow anatomical

Received September 11, 2019; Revised February 11, 2020; Accepted February 13, 2020 *Correspondence to : Prof. Micena Roberta Miranda Alves e Silva

Department of Morphology, Biological Sciences Institute, Federal University of Minas Gerais, Av. Antônio Carlos, 6627 - 31.270-901, Belo Horizonte, MG, Brazil Tel) 55-31-3409-3003, E-mail) micenarm@gmail.com structures, the use of a high-frequency $(7-20 \mathrm{MHz})$ linear US probe is ideal. ${ }^{2}$ Another important feature of the face is the extensive vascularization in the oral and maxillofacial region, which can be observed via the Doppler effect. ${ }^{3}$ Currently, US exams are not restricted to radiology clinics and have gradually become more prevalent in clinical settings through the use of portable equipment. The evaluation of anatomical structures can be done in real time, increasing the safety of the patient and the operator. Thus, procedures such as biopsies, aspirations, and injections can be performed with relatively high precision and accuracy. ${ }^{4}$

Filler injections in the facial areas have become more well-known in recent years due to the emergence of new technologies and formulations of the products used. How- 
ever, an important precaution concerning the stomatognathic system relates to its arrangement of esthetically and functionally important structures in close proximity to each other. ${ }^{5}$ Facial fillers are synthetic materials that are used in soft facial tissues for esthetic purposes and represent an alternative to invasive surgery. One widely-used filler is hyaluronic acid (HA). HA offers multiple benefits, including high biocompatibility, ideal physiochemical properties, and high levels of biosafety when injected properly. ${ }^{6}$ Although technological advances have made HA fillers more insoluble, resulting in a longer half-life, they are still degraded and absorbed in vivo and may exhibit different patterns of diffusion and distribution around the injection site over time. US is an effective way to anatomically evaluate the area of HA injection and to monitor the dynamics of the material in the surrounding tissue. Through US, it is possible to verify the locations of and relationships between important structures, such as vessels, muscles, and glands - which may influence the injection procedure - in addition to monitoring the movement of the filler through the tissue. $^{7}$

\section{Materials and Methods}

Two patients who underwent HA injections for esthetic purposes were followed in this study. The individuals did not have any health conditions that would preclude the execution of the procedures. A high-frequency (broadband with an array of $8.0 \mathrm{MHz}$ ) portable US device with a linear probe (Vscan with Dual Probe; GE Healthcare, Wauwatosa, WI, USA) was used to evaluate the areas of HA application and to monitor the injection sites over the months following the injection. The HA injection procedures were guided by US, and it was possible to observe both the injector position between the relevant tissues and the process of HA deposition. The US probe was positioned transversely in both cases, and a scan was performed of the region of interest to obtain information regarding nearby structures. All US examinations were performed by a single operator with extensive experience in the field, at the same location and with the same facial position of the probe for a given patient, while the operator constantly monitored the Doppler mode. The device specifications allowed the tissue to be viewed to a depth of $4 \mathrm{~cm}$, and excessive compression was not required in any of the examinations performed in this study.

The area of injection differed for the 2 patients, as did the HA filler used. In the first patient (a 57-year-old woman), the Perfecta ${ }^{\circledR}$ Subskin (Sinclair Pharma, Paris, France) filler was injected into the bilateral jaw angle region at a concentration of $20 \mathrm{mg} / \mathrm{mL}$. This filler is classified as biphasic and has a heterogeneous composition consisting of cross-linked HA particles in a carrier of non-cross-linked HA particles. ${ }^{8}$ The procedure was recommended because sagging jowls in the posterior jaw resulted in an aged appearance. Through the filler injections, we sought to promote the lifting and definition of tissue angles in this region.

By contrast, in the second patient (a 30-year-old woman), the Rennova ${ }^{\circledR}$ Fill (Croma GMBH, Leobendorf, Austria) filler was injected into the bilateral zygomatic region at a concentration of $23 \mathrm{mg} / \mathrm{mL}$. This filler is classified as monophasic, as it consists of a homogeneous gel composed of a mixture of long- and short-chain cross-linked HA. ${ }^{8}$ In this case, the aim of the filler injection was to highlight the zygomatic arch in order to alter the proportions between the middle and lower third of the face. Emphasizing the zygomatic arch creates a thinner and more delineated facial profile. It should be noted that the choice of filler was made in consideration of the facial dynamics and the rheological characteristics of HA. ${ }^{9}$ All injection procedures were performed by the same clinician.

In the first case, the injection was performed in the region of the jaw angle through a $22-\mathrm{G}$ cannula $(0.70$ $\mathrm{mm} \times 50 \mathrm{~mm}$ ), with an access point anterior to where the material was deposited. The use of a cannula was indicated due to the potential for use of a retrograde linear threading technique, the relatively high degree of cross-linking of the product, and the volume of material applied $(1.5 \mathrm{~mL}$ on each side). In addition, the region accessed by the cannula contains thicker tissues, provides more precise filling and thereby protects arterial branches, and reduces the possibility of trauma to the site. ${ }^{10}$ The cannula access point was approximately $3 \mathrm{~cm}$ anterior to the injection site, and the cannula was inserted into the subcutaneous region just below the subcutaneous adipose facial tissue.

By contrast, in the other case, a 27-G needle with a 0.5inch thin wall $(0.4 \mathrm{~mm} \times 12 \mathrm{~mm})$ was used to make the injection directly in the zygomatic region, $2 \mathrm{~mm}$ above the bone and between the muscle bundles. In this case, the use of the needle was justified by the technique used and the relatively low degree of crosslinking and density of the product, in addition to the planned placement of the HA filler in a deeper layer than in the first case. ${ }^{10}$ After the bilateral injection of approximately $0.5 \mathrm{~mL}$ of filler, the skin of each region was massaged to provide the necessary contour.

The cases were evaluated at 4 separate times - the moment of injection $\left(\mathrm{T}_{0}\right)$ and 24 hours, 30 days, and 180 days 
after injection $\left(T_{1}, T_{30}\right.$, and $T_{180}$, respectively $)$ - in order to monitor the filler movement.

To ensure the proper acquisition of the images in the region of the mandibular angle, we identified the subcutaneous facial adipose tissue, the parotid gland, the angle of the mandible, and the masseter muscle. In the zygomatic region, we attempted to identify both the masseter and the zygomatic bone.

\section{Results}

In US, hard tissues hinder the passage of US waves and produce a strong echo, as evidenced by hyperechoic margins. In contrast, soft tissues offer less resistance to waves and appear hypoechoic in intensity. In vivo, a region of HA filler appears as a hypoechoic delimited structure of low intensity or relatively low echogenicity (i.e., darker), as opposed to areas of edema, which appear as hypoechoic areas without defined limits. ${ }^{11}$ The tonality or darkness of HA depends, among other factors, on the degree of viscosity and density of the product, but HA can easily be differentiated from anatomical structures by its distinct boundaries with surrounding soft tissues. ${ }^{12}$ For locating and differentiating $\mathrm{HA}$, it is also helpful for the operator to have thorough knowledge regarding US techniques and the anatomy of the region.

In both cases in the present study, the US imaging performed before the procedure showed no tissue changes that would preclude the injection of the material. The invasion of vital structures, such as blood vessels, was avoided thanks to the US-guided procedure with Doppler imaging.
The color Doppler mode was also used after the injection to assess whether vascular damage had occurred. The HA filler was evident at all evaluated time points. The changes noted on US between the time points occurred due to the migration and shape changes of the filler material. However, these changes had no clinical impact on esthetics.

\section{Patient 1}

The area of the angle of the mandible was scanned via US, and the mandible bone, masseter muscle, parotid parenchyma, and subcutaneous tissue were identified (Fig. 1). HA injection through the cannula could be observed at $\mathrm{T}_{0}$, as could the extent of injector insertion into the tissue (Fig. 2A). Well-defined hypoechoic pockets of HA were formed and proved compatible with both the cannula path and the frequency of injection by the clinician, as observed 24 hours after the procedure at $\mathrm{T}_{1}$ (Fig. 2B). In the subsequent examinations, the pockets still exhibited a delimited character, but had spread to a position posterior to the point of their application, as observed at $\mathrm{T}_{30}$ (Fig. 2C). This movement was restricted to the anteroposterior direction, and no change in depth was detected; the filler remained in the sub-fat region where it was originally applied, as observed 180 days after filler injection (Fig. 2D). Interestingly, the filler presented as several circumscribed hypoechoic areas, arranged linearly between the subcutaneous screen and the masseter and lying horizontally in the same plane and superficially to the masseter muscle. Another important observation was the absence of vascular compression by the filler, as evaluated by US in Doppler mode; therefore, no ischemia or tissue necrosis was observed clinically.
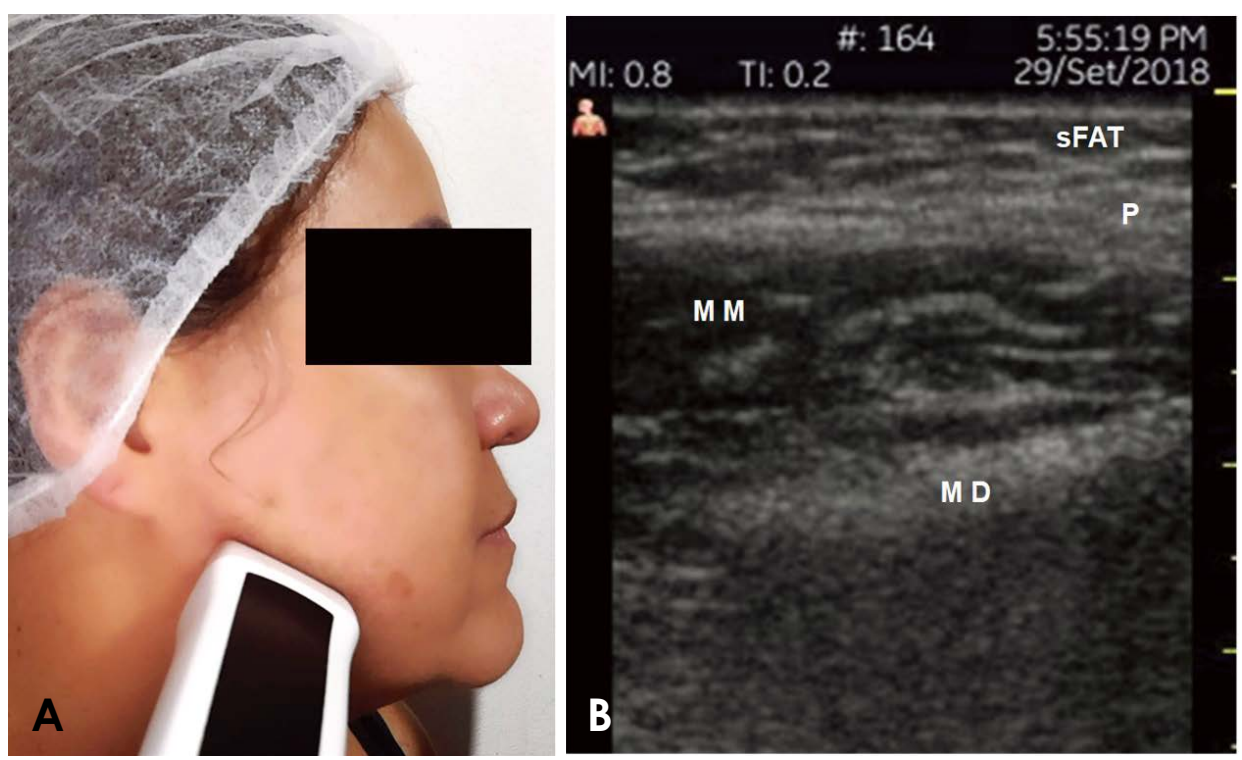

Fig. 1. Images of guided filling in the right angle of the mandibular angle (MD). A. Positioning of the transducer in the region of interest prior to filling. B. An ultrasound image taken before the introduction of the cannula, on which subcutaneous facial adipose tissue (sFAT) and the masseter muscle (MM) can be observed. P: parotid gland. 

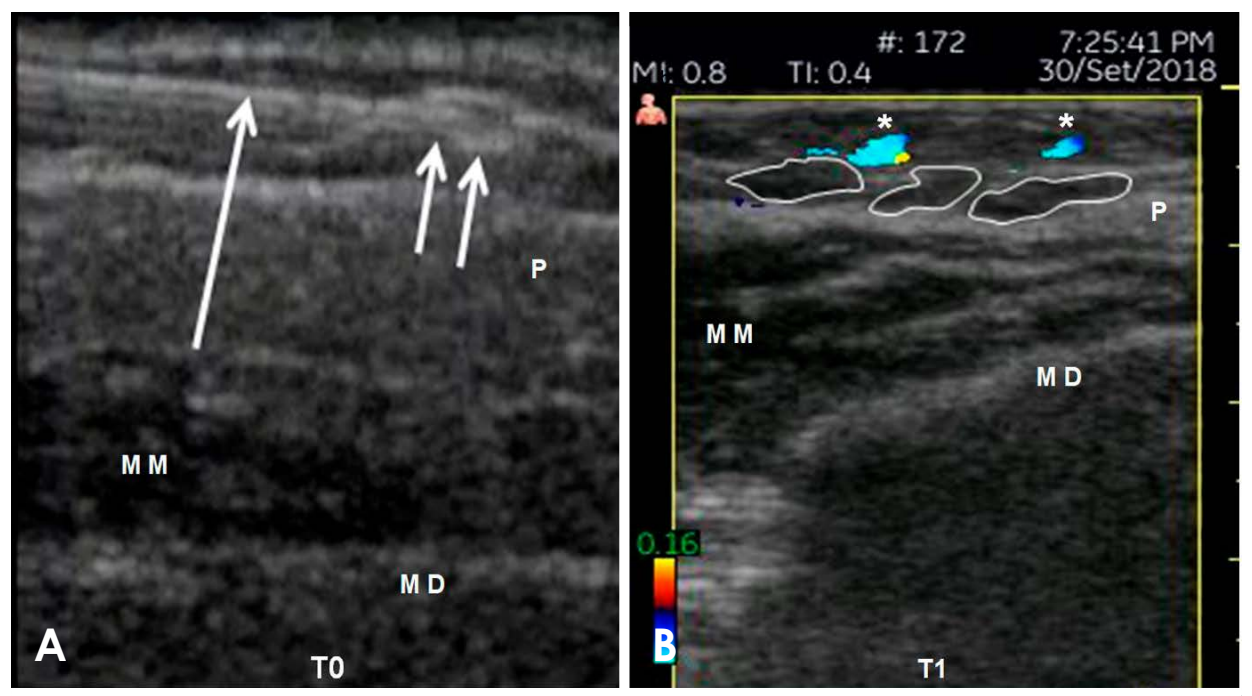

Fig. 2. Ultrasound images of the filling material in the right mandibular angle (MD). A. View of the cannula at the moment of injection $\left(\mathrm{T}_{0}\right)$ (larger arrow) and secure placement of the filler in the region (2 smaller arrows). B-D. Hypoechoic areas clearly corresponding to the filler. B. An image taken 24 hours after the procedure $\left(\mathrm{T}_{1}\right)$. C. An image taken 30 days after the procedure $\left(\mathrm{T}_{30}\right)$. D. An image taken 180 days after the procedure $\left(\mathrm{T}_{180}\right)$. Blood vessels (*) are identified using Doppler imaging. MM: masseter muscle, P: parotid gland.
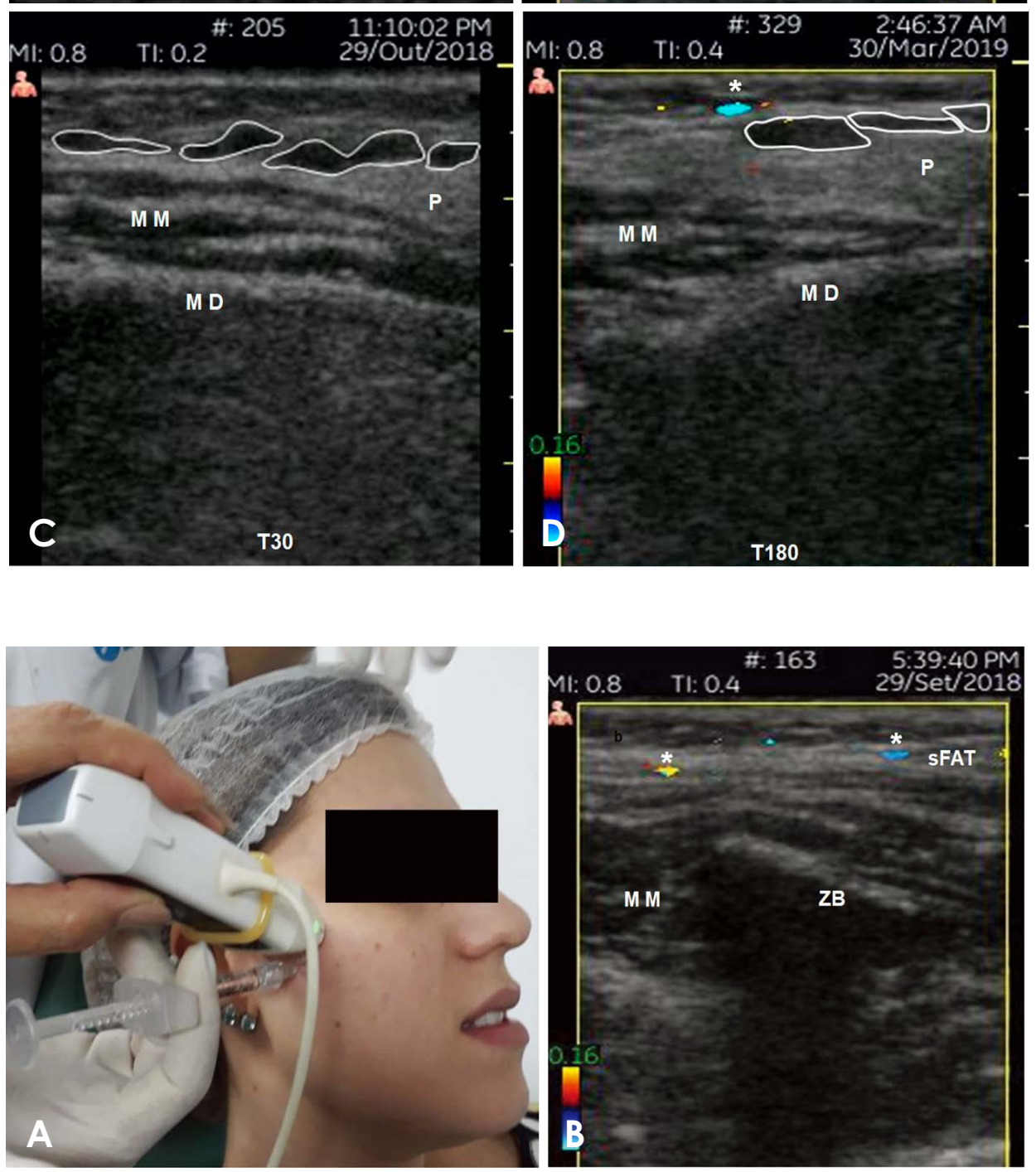

Fig. 3. Images of ulrasound-guided filler injection in the right zygomatic region. A. Positioning of the transducer and needle in the region of interest before filler injection. B. An ultrasound image taken before the introduction of the needle, showing the subcutaneous facial adipose tissue (sFAT), hyperechoic zygomatic bone (ZB), and hypoechoic masseter muscle (MM). Blood vessels (*) are identified using Doppler imaging.

\section{Patient 2}

The zygomatic region was scanned to visualize the anatomical structures (Fig. 3A). The region was observed to have thin subcutaneous tissue, most of which was occupied by muscle. The masseter muscle and the zygomatic bone were easily identified (Fig. 3B). At the moment of appli- 

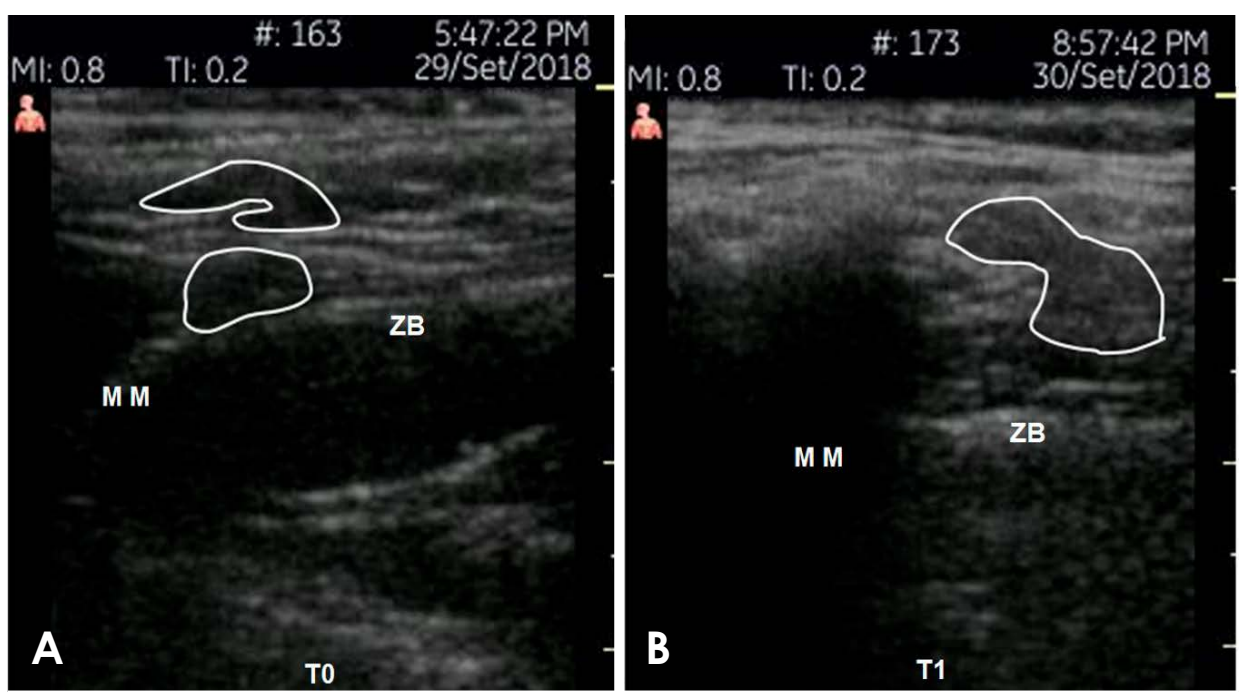

Fig. 4. Ultrasound images of the filling material in the right zygomatic region. A. View of the filler at the moment of injection $\left(\mathrm{T}_{0}\right)$ in the region (hypoechoic areas). B-D. Hypoechoic areas clearly corresponding to the filler. B. An image taken 24 hours after the procedure $\left(T_{1}\right)$. C. An image taken 30 days after the procedure $\left(\mathrm{T}_{30}\right)$. D. An image taken 180 days after the procedure $\left(T_{180}\right)$. Blood vessels (*) are identified using Doppler imaging. MM: masseter muscle, ZB: zygomatic bone.
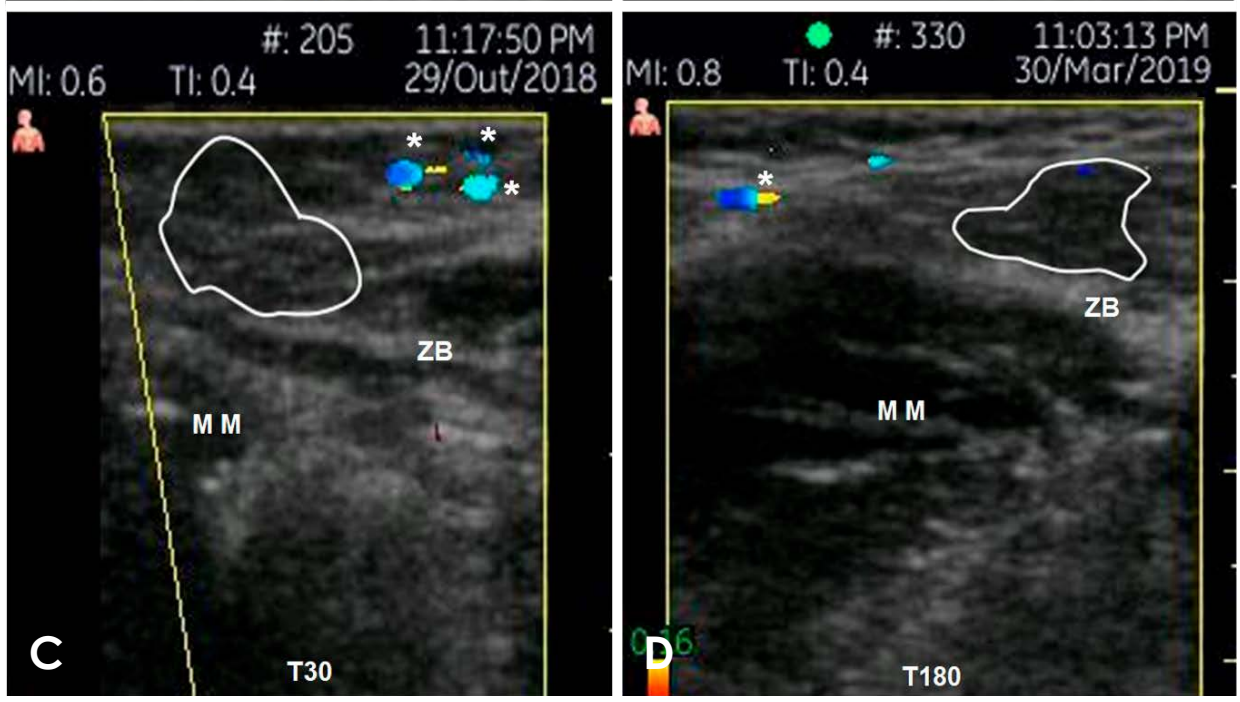

cation $\left(\mathrm{T}_{0}\right), 2$ well-delimited circumscribed pockets were visible (Fig. 4A), but these were less hypoechoic than those seen in case 1 . In the subsequent US exams, we observed the fusion of and discrete changes in the shape of the pockets, resembling bubbles, which continued to deepen toward the zygomatic bone over time (Figs. 4B-D). As reported for case 1, no vascular complications were observed. However, unlike in case 1 , the filler in the zygomatic region showed no change in its anteroposterior distribution over time.

\section{Discussion}

US with Doppler mode is an imaging modality already widely used in the medical field to evaluate structures throughout the body, but its use in the facial region is still infrequent. The technological advent of high-frequency, high-definition, and portable devices caused US to become more widespread among professionals of various special- ties, especially those who work with the facial region and the stomatognathic system. ${ }^{13}$ In addition, although cosmetic procedures are gaining importance due to the large number of procedures performed today, the literature indicates that US is still not part of the relevant protocols. However, US could assist in the pre-procedural diagnostic evaluation, the follow-up, and the evaluation of post-procedural complications. The use of facial fillers has come to clinicians' attention in recent years as a result of advances in the techniques and products used for orofacial harmonization. Although temporary, HA has been shown to be an outstanding filler due to its high biocompatibility and safety. Even so, little is understood regarding its dynamics and relationship with tissues in vivo over time. ${ }^{14}$ With this in mind, this work sought to demonstrate the applicability of US as a highly useful auxiliary tool in esthetic procedures performed in facial regions.

In the cases presented in this article, we used US exam- 
inations to make an imaging diagnosis and to aid in clinical examinations. The US images allowed us to precisely visualize the HA injection site and adjacent structures beyond the material itself, ${ }^{15}$ ensuring greater safety for the professional and minimizing complications for the patient. In patient 1 , the application site was superficial, located just below the facial fat and above the masseter muscle and part of the parenchyma of the parotid gland. Because the HA was applied at well-defined tissue interfaces, the image generated of the filler was well-defined and dark, with no interference from other structures. ${ }^{16}$ The position of the material was observed to be posterior to the place of application, fully above the parotid parenchyma, at $\mathrm{T}_{180}$.

In patient 2, different US features were observed due to differences in the material used and the region evaluated. HA was applied to augment the patient's thin subcutaneous tissue through an injection between the muscle bundles. A more hyperechoic filler image was generated than in the previous case, likely due to the interference from the region's structures or differences in the degree of cross-linking between the materials. This distinct clinical behavior was also observed by Goh et al. when studying the use of 2 types of HA gels as fillers in the periocular region; in that study, different US features were reported due to differences in chemical processing technologies. In the present study, the bubble conformation of the HA observed in the second case (which differed from the pattern of the HA filler in the first case) may have been due to the influence of facial muscle movement in the zygomatic region. ${ }^{9,12}$ However, despite the difference in the type of HA injected, the material could be easily observed in both cases, as could its proximity to other structures.

Because the procedure was guided by US in real time, no complications were observed, as would have been expected if the injection had been administered in an inappropriate place. It is emphasized that the differentiation between blood vessels and other surrounding structures enabled by Doppler-mode US prevented vascular compression by the filler. This study also shows that, for at least 180 days, US can be used to detect HA filler and to assess its condition in vivo.

In conclusion, the present study reinforces the importance of US as a tool to guide the clinician in the placement of filler in the facial region, as well as to monitor its clinical manifestations. It is necessary to emphasize that, in order to obtain and interpret US images, a qualified professional with extensive anatomical knowledge must conduct the evaluations.

\section{References}

1. Sahlani L, Thompson L, Vira A, Panchal AR. Bedside ultrasound procedures: musculoskeletal and non-musculoskeletal. Eur J Trauma Emerg Surg 2016; 42: 127-38.

2. Young SR, Bolton PA, Downie J. Use of high-frequency ultrasound in the assessment of injectable dermal fillers. Skin Res Technol 2008; 14: 320-3.

3. Abdelsalam TA, Amer ME, Mahrous A, Abdelkader M. Evaluation of oral and maxilofacial swellings using ultrasonographic features. Imaging Sci Dent 2019; 49: 201-8.

4. Scholten HJ, Pourtaherian A, Mihajlovic N, Korsten HH, A Bouwman R. Improving needle tip identification during ultrasound-guided procedures in anaesthetic practice. Anaesthesia 2017; 72: 889-904.

5. Signorini M, Liew S, Sundaram H, De Boulle KL, Goodman GJ, Monheit G, et al. Global aesthetics consensus: avoidance and management of complications from hyaluronic acid fillers-evidence- and opinion-based review and consensus recommendations. Plast Reconstr Surg 2016; 137: 961e-71.

6. Attenello N, Maas C. Injectable fillers: review of material and properties. Facial Plast Surg 2015; 31: 29-34.

7. Schelke LW, Decates TS, Velthuis PJ. Ultrasound to improve the safety of hyaluronic acid filler treatments. J Cosmet Dermatol 2018; 17: 1019-24.

8. Pierre S, Liew S, Bernardin A. Basics of dermal filler rheology. Dermatol Surg 2015; 41 Suppl 1: S120-6.

9. Michaud T. Rheology of hyaluronic acid and dynamic facial rejuvenation: topographical specificities. J Cosmet Dermatol 2018; 17: 736-43.

10. Pavicic T, Frank K, Erlbacher K, Neuner R, Targosinski S, Schenck T, et al. Precision in dermal filling: a comparison between needle and cannula when using soft tissue fillers. J Drugs Dermatol 2017; 16: 866-72.

11. Josse G, Haftek M, Gensanne D, Turlier V, Mas A, Lagarde JM, et al. Follow up study of dermal hyaluronic acid injection by high frequency ultrasound and magnetic resonance imaging. J Dermatol Sci 2010; 57: 214-6.

12. Goh AS, Kohn JC, Rootman DB, Lin JL, Goldberg RA. Hyaluronic acid gel distribution pattern in periocular area with high-resolution ultrasound imaging. Aesthetic Surg J 2014; 34 : 510-5.

13. Schelke LW, Van Den Elzen HJ, Erkamp PP, Neumann HA. Use of ultrasound to provide overall information on facial fillers and surrounding tissue. Dermatol Surg 2010; 36 Suppl 3: 184351.

14. Vent J, Lefarth F, Massing T, Angerstein W. Do you know where your fillers go? An ultrastructural investigation of the lips. Clin Cosmet Investig Dermatol 2014; 7: 191-9.

15. Wortsman X. Identification and complications of cosmetic fillers: sonography first. J Ultrasound Med 2015; 34: 1163-72.

16. Greene JJ, Sidle DM. The hyaluronic acid fillers: current understanding of the tissue device interface. Facial Plast Surg Clin North Am 2015; 23: 423-32. 